Service social

\title{
L'intervention auprès d'adolescents : un lieu favorable au développement de la prévention primaire
}

\section{Monique Tessier}

Volume 35, numéro 3, 1986

Les jeunes et le travail social

URI : https://id.erudit.org/iderudit/706314ar

DOI : https://doi.org/10.7202/706314ar

Aller au sommaire du numéro

Éditeur(s)

École de service social de l'Université Laval

ISSN

1708-1734 (numérique)

Découvrir la revue

Citer cet article

Tessier, M. (1986). L'intervention auprès d'adolescents : un lieu favorable au développement de la prévention primaire. Service social, 35(3), 304-315. https://doi.org/10.7202/706314ar
Résumé de l'article

Suggérant des approches nouvelles dans la pratique du service social auprès d'adolescents, l'auteure nous présente les principes théoriques de l'entraide, formule qu'elle privilégie auprès des jeunes ; elle fait aussi état d'une recherche québécoise qui a encadré deux expériences simultanées de cette nature auprès d'adolescents en milieu scolaire. 
TESSIER, Monique, travailleuse sociale au C.L.S.C. Villeray, à Montréal.

\section{L'intervention auprès d'adolescents : un lieu favorable au développement de la prévention primaire}

\section{Monique Tessier}

La reconnaissance de la sexualité adolescente est un phénomène fort nouveau dans le champ des sciences humaines. Formellement interdit, nié, même réprimé pendant longtemps, le comportement sexuel des adolescents * a, depuis les dix dernières années, au Québec, donné naissance à une problématique nouvelle : un nombre croissant de jeunes confrontés à des grossesses non désirées ou encore atteints de maladies transmissibles sexuellement (M.T.S.). Non plus limitée à des cas isolés, cette situation prend de l'ampleur et apparaît comme une réalité ayant des implications non seulement médicales, mais aussi psychologiques et sociales. Pour répondre aux besoins, dans un premier temps, on développe des programmes et des cliniques jeunesse dans les centres locaux de services communautaires (C.L.S.C.), et des cliniques pour adolescents dans quelques centres hospitaliers. En attendant un programme officiel, plusieurs écoles, tant primaires que secondaires, expérimentent des "avant-projets" en matière d'éducation sexuelle. Les départements de santé communautaire s'intéressent aussi à cette question ; quelques-uns ont procédé à une revue de la littérature sur la santé des adolescents (Ayotte et Roy, 1984), et les grossesses adolescentes (Filion et Thébault, 1984), et ont proposé de nouvelles stratégies d'intervention.

Tous ces établissements ont grandement contribué à la reconnaissance de la sexualité adolescente et au développement des connaissances dans le domaine.

La distribution de services cliniques implique souvent des collaborations entre intervenants et nous permet de constater que la sexualité adolescente préoccupe aussi d'autres milieux : les centres de services sociaux, avec l'application de la Loi sur la protection de la jeunesse, les 
centres d'accueil spécialisés auprès d'adolescents, les animateurs de maisons de jeunes, les enseignants et les professionnels du milieu scolaire.

Récemment, des études réalisées par le Conseil des affaires sociales et de la famille (1984) et par le Comité de la santé mentale du Québec (1985) faisaient état des priorités en matière de santé physique et mentale chez les adolescents ; les auteurs y suggèrent des approches qui nous invitent à situer la sexualité adolescente dans un cadre plus large et à développer des actions à travers des formules basées sur la concertation. Le présent article se veut une contribution dans ce sens : il suggère une approche nouvelle en présentant une vision élargie des besoins des adolescents, des modèles théoriques qui s'intéressent à la prévention, et livre des résultats d'études qui réfèrent à des expériences récentes.

\section{Problématique}

Une étude effectuée à Calgary (Meikle, Pearce, Peitchinis et Pysh, 1981) auprès de 809 adolescents âgés de 13 à 18 ans montre que le tiers d'entre eux avaient eu des relations sexuelles au moment de l'enquête ; on retrouve la même proportion dans l'étude effectuée par Camille Messier (1981) auprès d'une population fréquentant une école secondaire de Montréal. La moyenne d'âge lors des premières relations sexuelles varie entre 14 et 16 ans. Au Québec, en 1980, plus de 9000 adolescentes de moins de 20 ans devenaient enceintes. Un peu plus de 5000 d'entre elles donnaient naissance à leur enfant (Statistique Canada, 1982) tandis qu'environ 4000 obtenaient un avortement (R.A.M.Q., 1982).

En 1980, 50\% des cas de gonorrhée déclarés au Québec se retrouvaient chez les 15 à 19 ans. Le rapport sur la santé des Québécois (Conseil des affaires sociales et de la famille, (1983) rappelle que, lorsqu'elle n'est pas traitée, la gonorrhée peut entraîner l'infertilité chez l'homme comme chez la femme. Un des problèmes reliés au traitement et au contrôle de cette maladie est le fait qu'elle est souvent asymptomatique.

Le dernier Congrès international sur les maladies transmissibles sexuellement, tenu à Montréal en juin dernier (L'Actualité médicale, 1984), a permis de faire le point sur la gravité des complications dues à ces maladies, chez les jeunes en particulier. On estime qu'en l'an 2000 , une femme sur deux aura été atteinte avant l'âge de 30 ans de salpingite surtout causée par le chlamydia ou la gonorrhée. On constate actuellement que $8 \%$ à $10 \%$ des femmes sexuellement actives, entre 17 et 25 ans, 
sont atteintes de chlamydia, maladie semblable à la gonorrhée. Dans un relevé de cent dossiers actifs au cours de l'année 1984 à la Clinique des jeunes Saint-Denis, on observe que $5 \%$ des adolescents ont été traités pour gonorrhée et/ou chlamydia.

Récemment, l'auteure de cet article publiait une étude qui visait à identifier les motifs de consultation des adolescentes qui se présentent dans un centre local de services communautaires (C.L.S.C.) et à mieux cerner leurs besoins, tant implicites qu'explicites (Tessier, 1985). La recherche visait aussi à recueillir des données en vue de développer des actions préventives, plus particulièrement orientées vers l'utilisation d'adolescents comme multiplicateurs auprès des autres.

Les résultats nous apprennent que $40 \%$ des adolescentes qui consultent soupçonnent déjà une grossesse lors de leur première visite. De plus, $\mathbf{8 0} \%$ des sujets qui se sont présentés durant la période d'expérimentation ont déclaré avoir déjà eu une ou plusieurs relations sexuelles. Une partie importante de ce groupe peut aussi être considérée à risque, face à une grossesse non désirée, si l'on réfère à l'ensemble des études qui font état de la faible utilisation des contraceptifs durant la première année des activités sexuelles des adolescents (Cobliner, 1974 ; Meikle et al., 1981 ; Zelnik et Kantner, 1980).

Le $D^{r}$ Nicole Onetto (1982), dans une étude effectuée au Centre hospitalier Sainte-Justine auprès de cent adolescentes, constate que seulement $24 \%$ d'entre elles ont démontré une fidélité parfaite à l'endroit du programme, c'est-à-dire qu'elles ont respecté leurs rendezvous sans que l'infirmière ait eu à téléphoner et qu'elles ont utilisé adéquatement leur méthode de contraception sans aucune interruption durant les douze mois d'expérimentation. Ces chiffres nous démontrent que des services cliniques, même assurés par un personnel compétent et au moyen d'approches adaptées aux adolescents, ne peuvent à eux seuls solutionner le problème des grossesses non désirées.

Le $D^{r}$ Jean Wilkins (1985) a été l'un des premiers, au Québec, à traiter de la présence de composantes multiples expliquant le comportement sexuel et contraceptif de l'adolescent; ses écrits soulignent l'interaction entre les composantes biologique, psychologique et sociologique. L'étude effectuée à la Clinique des jeunes Saint-Denis confirme ces résultats : les sujets ont reconnu que leurs problèmes psychologiques les plus importants sont la communication avec les parents, les conflits personnels, la présence de grandes peurs et de cauchemars. Dans le domaine de la santé physique, c'est la nervosité qui, après la crainte d'une grossesse, est apparue comme le problème le plus important, suivi des maux de tête et des difficultés reliées au sommeil. Précisons que les problèmes se présentent dans le même ordre, que les adolescentes 
soient actives ou non sexuellement. On peut donc affirmer, sans crainte de se tromper, que lorsqu'un adolescent fait appel à des services en sexualité et en planification des naissances, il est souvent confronté à des difficultés diverses qu'il abordera d'ailleurs rarement de lui-même. L'étude effectuée sur la santé mentale des adolescents par le D.S.C. du Centre hospitalier de l'Université Laval (Ayotte et Roy, 1984) présente des résultats semblables. En effet les relations parents-adolescents ainsi que les comportements associés à la drogue figurent au premier rang des difficultés rencontrées par les jeunes. Les problèmes que l'on associe aux parents réfèrent surtout aux conséquences du divorce, aux difficultés de communication, au manque d'amour, de compréhension et d'attention de leur part. Les intervenants consultés dans cette même étude perçoivent aussi que les jeunes auprès desquels ils travaillent, qu'ils soient suicidaires ou aux prises avec une grossesse non désirée, éprouvent tous des difficultés avec leurs parents, les amis ou l'école. On ne s'étonnera donc pas des résultats contenus dans le bilan sur la santé des Québécois, lequel présente les troubles mentaux comme responsables, chez les 15-24 ans, du plus grand nombre de journées d'hospitalisation chez les garçons alors qu'ils viennent au second rang chez les filles du même âge, juste après les problèmes de santé liés à la grossesse et à l'accouchement.

Mais vous me direz que nous sommes en train d'oublier la sexualité adolescente dans tout cela. Allons donc voir comment ça se passe dans la réalité quotidienne. Récemment, une infirmière d'une école secondaire de la région de Montréal recevait à son bureau une adolescente de 12 ans, shootée à l'acide, comme on dit dans le milieu, gelée sous l'effet du haschich, ayant absorbé du "béta-dix"; l'adolescente, déjà active sexuellement, ne demandait qu'à être référée pour fin de contraception en mentionnant évidemment que ses parents ne devaient pas être informés de sa démarche. De quel problème s'agit-il au juste? Sexualité précoce ? Risque de grossesse ? Dépendance face à la drogue ? Conflits personnels importants? Communications parents-adolescents? Pression exercée par les amis? Ou encore un problème relié à l'existence de pushers dans l'école? Ces questions, telles qu'elles se posent dans notre quotidien, prouvent, qu'entre la santé physique et la santé mentale, il n'y a vraiment qu'un pas!

\section{Cadre théorique}

La notion de santé s'est transformée au cours des années; au lieu de n'y voir que l'absence de la maladie, elle apparaît maintenant 
comme "le résultat de l'adaptation de l'individu aux sollicitations de son environnement» (C.A.S.F., 1984).

L'intervention qui tient compte du caractère dynamique de la santé exige des actions à des moments différents et à trois niveaux de prévention:

- primaire, qui vise à empêcher l'apparition de symptômes et à favoriser le prolongement d'un état de santé optimal (informations, groupes d'échanges entre adolescents) (Saucier et Steinberg, 1979) ;

- secondaire, où l'on intervient rapidement après l'apparition des symptômes (par exemple: consultations auprès d'adolescentes déjà actives sexuellement et n'utilisant aucune méthode contraceptive) ;

- tertiaire, où l'on intervient pour soulager et traiter des conditions chroniques irréversibles (par exemple: interruption de grossesse).

Rosenstock (1974) explique qu'un individu ne peut adopter un comportement préventif qu'à certaines conditions :

- il doit être conscient des risques et des dangers que comporte sa conduite ;

- il faut qu'il perçoive des gains à l'adoption d'une nouvelle conduite et qu'il puisse en évaluer les coûts ;

- finalement, il semble que, même s'il perçoit les risques et les gains possibles et qu'il est conscient des coûts d'une action préventive, l'individu a besoin d'une poussée supplémentaire pour agir, un événement incitateur, comme des formes de publicité, de pressions, etc.

Rokeach (1973), pour sa part, soutient que les valeurs sont modifiables chez les individus et que, si l'on parvient à en modifier ou en changer une, il en résulte des changements systématiques dans les attitudes et les comportements.

Le développement des compétences constitue une des formes d'application de la prévention primaire. On définit la compétence comme une capacité, une habileté qui permet à l'individu de réussir dans l'exercice d'une fonction ou dans l'exécution d'une tâche donnée (Comité de la santé mentale, 1985a). Ayotte et Roy (1985) présentent diverses stratégies qui visent cet objectif : celle du support social (Cassel, 1974) qui suggère, non seulement de travailler à réduire les facteurs qui expliquent les problèmes rencontrés (par exemple, les résistances à la contraception), mais aussi au renforcement de ceux qui 
les diminuent (comme le développement de l'estime de soi, de meilleures relations parents-adolescents, etc.).

Le modèle écologique est aussi une stratégie qui contribue au développement des compétences. Camil Bouchard (cité dans : J. Arseneau et al, 1983) le compare au modèle médical pour en faciliter la compréhension. Alors que, selon ce dernier modèle le problème psychologique relève de la maladie, le modèle écologique y verra une difficulté d'adaptation. Dans le premier cas, l'intervention sera de type individuel et une thérapie verra à éliminer la pathologie. Dans le second, le problème résultant d'une interaction entre l'individu et son environnement, on interviendra aux deux niveaux. La prévention sera de type secondaire et tertiaire dans le cas du modèle médical (dépistage, meilleure accessibilité, suivi plus serré) et elle sera de type primaire dans le modèle écologique (développement des compétences personnelles en même temps que développement d'environnements adéquats pour l'individu).

Ces approches préventives visant le renforcement du support social et le développement des compétences suggèrent la reconnaissance et la promotion du rôle joué par les non-professionnels. Dans une étude citée antérieurement (Tessier, 1985), l'auteure a démontré l'importance de l'influence que les adolescents exercent les uns sur les autres : dans $63 \%$ des cas, ce sont les amies qui ont référé les sujets à la clinique. Ce sont aussi elles qui sont jugées par la très grande majorité des sujets comme les interlocutrices privilégiées en matière de sexualité et de contraception. Invitées à se prononcer sur l'intérêt à consulter un "pair aidant " en situation de besoin, elles ont répondu affirmativement dans une proportion de $75 \%$; de plus, c'est dans une proportion de $80 \%$ qu'elles ont manifesté un intérêt à exercer elles-mêmes un rôle d'aidant.

Une recherche publiée par le Département de santé communautaire du Centre hospitalier de l'Université Laval présente les résultats d'expérimentations réalisées simultanément à Québec et à Montréal auprès de jeunes multiplicateurs de niveau secondaire. Subventionnée par le Ministère de la santé et des services sociaux, cette étude vise à « mettre en lumière les processus interactifs par lesquels les groupes de pairs en viennent à aider efficacement d'autres jeunes" (Roy, 1986: 1). MarieRenée Roy souligne toutefois la difficulté et le paradoxe de l'évaluation des activités d'entraide : difficile, puisque l'auteure constate que l'aide individuelle entre jeunes se situe presque exclusivement dans le domaine de l'informel, donc apparaît peu mesurable ; paradoxale, puisque c'est "le propre de l'aide naturelle que de s'opérer à travers les mécanismes sociaux habituels et non à travers une relation formelle aidant-aidé " 
(Roy, 1986 : 17). Le rapport révèle toutefois quelques indices utiles à l'évaluation des deux projets. Interrogés individuellement à la fin de l'expérimentation, les jeunes d'un des groupes ont souligné que leur participation à l'organisation d'une semaine sur la nutrition dans l'école avait resserré l'esprit d'équipe. Cela leur avait aussi permis de réaliser qu'ils pouvaient jouer un rôle actif dans leur école et ce, à travers des relations significatives avec des adultes. L'auteure souligne le fait que, des deux activités planifiées par les jeunes dans une des écoles, seule celle où un adulte du milieu s'est impliqué avec eux s'est réalisée. Les changements survenus dans le rôle de l'infirmière en milieu scolaire, durant et à la suite des expériences, permettent aussi d'évaluer l'impact de ces actions dans ce contexte : elles se sont vu référer un plus grand nombre de jeunes, tant par les multiplicateurs que par les adultes du milieu, et les interventions requises furent surtout psychosociales.

Autre manifestation de l'impact de tels projets sur le milieu: l'année suivant l'étude du D.S.C., la direction d'une des écoles a intégré la formation à l'entraide dans l'ensemble des activités socio-culturelles proposées aux étudiants au début de chaque année. Cette initiative permet ainsi à deux enseignants de s'impliquer activement dans l'activité à l'intérieur de leur charge de travail, au rythme de une à deux périodes hebdomadaires de 75 minutes chacune. En l'intégrant dans son propre programme, l'école s'approprie progressivement cette activité née d'une concertation entre jeunes, école, C.L.S.C., D.S.C., parents et commission scolaire.

Une expérience, américaine cette fois, donne un autre aperçu de l'efficacité de l'utilisation de pairs adolescents et de l'intervention exercée sur le milieu. Dans L'école et la santé mentale, le Comité de la santé mentale (1985b) fait état d'un programme centré sur la drogue dans plusieurs écoles publiques de la ville de New York ; son évaluation a permis d'observer des résultats concluants suite à l'utilisation de stratégies visant le support social. Les mesures adoptées allaient de conseils donnés individuellement ou en groupes, à l'utilisation d'animateurs choisis par les élèves dans les groupes, à l'utilisation d'adultes significatifs tout en stimulant une participation de la communauté. Nowlis (1980) a observé qu'après un an, le nombre d'élèves signalés pour problèmes de drogues avait diminué de façon sensible alors qu'il avait augmenté dans le groupe de contrôle. L'absentéisme scolaire avait baissé de même que les bagarres et les injures pendant qu'on notait une augmentation dans le groupe contrôle. Les mêmes observations ont été faites concernant les résultats scolaires.

Nos milieux étant généralement inexpérimentés et peu préparés au développement de telles actions, les chances de succès de projets 
voués à la prévention primaire seront proportionnelles à l'importance qu'on accordera au respect de la suite chronologique des étapes définies par Tessier (1985) :

1. planification et concertation

2. organisation et programmation

3. opérationnalisation

4. évaluation

5. contrôle des résultats et feedback.

\section{Participation et concertation}

L'intervenant choisit, dans un premier temps, les modèles théoriques sur lesquels il veut appuyer son action. Il détermine le type d'intervention qu'il souhaite réaliser, puis met en place un mécanisme de concertation à partir duquel il associe les personnes et les organismes impliqués, incluant les bénéficiaires visés par le projet. Ensemble, les différentes parties identifient les besoins, à partir de consultations variées, de recherches sur le terrain et d'une revue de la littérature. Ils sont alors prêts à définir les objectifs.

\section{Organisation et programmation}

À cette étape, il faut se donner les moyens qui permettront d'évaluer les objectifs retenus. On définit alors le type d'activités qu'on veut mettre sur pied, le nombre et la durée des interventions. On choisit les personnes-ressources qui s'impliqueront dans la réalisation du projet, et on fixe le nombre de participants que l'on désire. Des résultats anticipés au niveau du comportement individuel et de l'impact souhaité sur le milieu faciliteront l'évaluation des objectifs. La programmation sera établie en fonction des besoins identifiés et des caractéristiques observées auprès de la population visée. Elle tiendra compte du milieu où se déroulera l'action (école, maison de jeunes, C.L.S.C., etc.), du type de participation recherchée chez les bénéficiaires, ainsi que du choix et du rôle des personnes-ressources.

\section{Opérationnalisation}

C'est le moment de la mise en marche du projet. Au fur et à mesure de son déroulement, les responsables évaluent son fonctionnement: ils s'assurent de son accessibilité réelle, sensibles au choix des lieux, de 
I'horaire, etc. Ils s'attardent aussi à la disponibilité et la compétence des personnes-ressources.

\section{4. Évaluation}

En se référant aux critères établis à l'étape de l'organisation et de la programmation, les intervenants cherchent à évaluer l'impact de leur projet sur les conditions de vie des participants et sur leur milieu de vie en général. Ils sont curieux d'observer si les concepts théoriques retenus ont circulé dans le milieu et si les besoins évalués au point de départ correspondaient bien aux besoins réels, tels qu'observés au cours de l'expérimentation. Il devient alors possible de juger du réalisme des objectifs poursuivis et de la pertinence des critères sélectionnés. L'évaluation permet aussi de découvrir la présence des personnes significatives dans leur milieu et leur relation avec les bénéficiaires du projet. C'est à cette étape qu'on évalue le dynamisme démontré par les participants durant l'expérience ainsi que la nature et la qualité de la contribution qu'ils y ont apportée.

\section{Contrôle des résultats et feedback}

Il est important de reconnaître qu'une intervention préventive s'inscrit dans un processus continu et ininterrompu. Ainsi, selon les résultats, elle donnera lieu à des changements dans le milieu et, possiblement même, à de nombreux programmes ou de nouvelles activités. À leur tour, ces initiatives auront un impact sur la qualité de vie des gens qui y vivent. Et le cycle se poursuit : planification, organisation, opérationnalisation, évaluation, contrôle de résultats, feedback, planification, etc.

Jusqu'à quel point ces modèles nous apparaissent-ils applicables pour la majorité d'entre nous, qui, convenons-en, avons été formés selon le modèle médical traditionnel. Dans son Avis, le Comité sur la santé mentale affirme "qu'une des bases de l'action préventive réside dans la capacité de saisir les questions dans son ensemble et dans la concertation de toutes les forces et ressources du milieu." (C.S.M., 1985a : 2.)

Dans cette optique, le document du Comité de la Commission des écoles catholiques de Montréal sur la qualité de vie des élèves du secondaire (Baulu, 1985) présente une critique et une redéfinition des stratégies qui utilisent les professionnels de l'école. On considère ainsi que la formule traditionnelle appelée « stratégie par problèmes » segmente 
la réalité des jeunes et risque de perdre de vue l'ensemble de la qualité de vie à l'école. Le rapport suggère plutôt le développement de " stratégies globales " qui verront à harmoniser ou à intégrer les différents programmes en une action coordonnée et efficace.

Pour sa part, Roy (1986: 25-32) dégage et commente sept principes théoriques applicables à l'intervention par les pairs et susceptibles de faciliter la mise sur pied de groupes de jeunes multiplicateurs :

1. deux motivations à respecter : soi et les autres

2. assurer une progression dans l'intervention : respecter l'évolution

3. donner un "cadre" aux activités du groupe

4. référer constamment au vécu des jeunes

5. mobiliser les jeunes dans l'action

6. I'animateur: un support, un guide, un facilitateur

7. y accorder du temps et de soi-même.

C'est grâce à sa proximité des expériences-terrain et à son implication dans les diverses étapes de la réalisation de projets que l'auteure a pu dégager de telles propositions; cet exemple illustre bien l'intérêt qu'ont les intervenants à articuler étroitement les processus de réalisation et d'encadrement de leurs projets!

\section{Conclusion}

Au Québec, l'intervention en matière de sexualité adolescente s'est jusqu'à maintenant beaucoup concentrée sur la solution des problèmes; par conséquent, on a favorisé le développement de services cliniques.

Parallèlement, on assiste à une recherche de solutions alternatives qui modifient le rôle des usagers et celui des intervenants. Cette ouverture donne lieu à des expériences novatrices (voir l'article suivant), où l'on met davantage l'accent sur les besoins et les ressources de l'individu que sur ses problèmes.

La faisabilité de ces actions, l'enthousiasme qu'elles génèrent et l'intérêt qu'y porte la recherche ne devraient-ils pas apparaître comme d'excellents précurseurs à des rôles nouveaux dans la pratique du service social?

\section{Note}

* La forme masculine sera utilisée tout au long de ce texte pour désigner aussi bien les filles que les garçons. 


\section{Références bibliographiques}

Arseneau, J., C. Bouchard, M. Bourgon, G. Goupil, J. Guay, F. Lavole et R. Perreault, Psychothérapies: Attention !, Québec, Québec Science éditeur, 1983.

Arotre, V. et M.-R. Ror, Santé mentale chez les adolescents : proposition d'un projet d'intervention, texte non publié, Québec, Département de santé communautaire du Centre hospitalier de l'Université Laval, 1985.

Ayotre, V. et M.-R. RoY, Santé des adolescents : bilan des études et perspectives d'intervention, Québec, Département de santé communautaire du Centre hospitalier de l'Université Laval, 1984.

BAULu, P., L'école et la santé mentale, état de la question de la santé mentale en milieu scolaire, document d'information adressé au Comité de la santé mentale du Québec pour la préparation d'un avis au ministre des Affaires sociales du Québec, Montréal, Commission des écoles catholiques de Montréal, 1985.

CASSEL, J., "Psychosocial processes and stress : Theoretical formulation, "International Journal of Health Services, vol. 4, no 3, 1974 : 471-482.

"Chlamydia trachomatis : cause de 45 à $60 \%$ des cas de salpingite", L'Actualité médicale, vol. 5, no 14, 1984.

Cobliner, W.G., " Pregnancy in the single adolescent girl, the role of cognitive functions ", Journal of Youth and Adolescence, vol. 3, no 1, 1974 : 17-28.

Comité de la santé mentale du Québec, La santé mentale des enfants et des adolescents. Vers une approche plus globale, Québec, Bibliothèque nationale du Québec, 1985a.

Comité de la santé mentale du Québec, L'école et la santé mentale, état de la question de la santé mentale en milieu scolaire, Québec, Bibliothèque nationale du Québec, $1985 \mathrm{~b}$.

Conseil des affaires sociales et de la famille, La santé des Québécois, Québec, Éditeur officiel du Québec, 1983.

Conseil des affaires sociales et de la famille, Objectif : santé. Rapport du Comité d'étude sur la promotion de la santé, Québec, Bibliothèque nationale du Québec, 1984.

Filion, G. et M. Thébautt, Grossesse et adolescence, revue de la littérature et éléments de problématique, Montréal, Département de santé communautaire du Centre hospitalier Saint-Luc, 1984.

Meikle, S., K. Pearce, J. Peitchinis et F. Pysh, An Investigation into the Sexual Attitudes, Knowledge and Behavior of Teenage School Students, Calgary, United Way of Calgary, 1981.

MEssier, C., La sexualité : vécu et opinions d'un groupe de jeunes, Montréal, Comité de la protection de la jeunesse, Ministère de la justice, 1981.

Nowus, H., La drogue démythifiée: drogue et éducation, $2 \mathrm{e}$ édition révisée, Paris, Presses de l'UNESCO, 1980.

Onetro, N., La contraception de l'adolescente et ses aspects spécifiques: une expérience canadienne, thèse de doctorat non publiée, Paris, Université René-Descartes, 1982. 
Régie de l'assurance-maladie du Québec, Statistiques annuelles, Québec, R.A.M.Q., 1982.

RoKeACH, M., The Nature of Human Values, New York, Free Press, 1973.

ROSENSTOCK, I.M., "The health belief model and preventive health behavior", dans M.H. Becker (éd.), The Health Belief Model and Personal Health Behavior, Thorofare (N.J.), C.B.S., 1974.

Roy, M.R., La dynamique des groupes de pairs aidants: réflexions à partir de deux projets pilote dans des écoles secondaires québécoises, Québec, Département de santé communautaire du Centre hospitalier de l'Université Laval, 1986.

SAUCIER, J.-F. et M. SteINBERG, Adolescents et prévention, (rapport préliminaire), Montréal, Université de Montréal, Département de psychiatrie, Section des sciences du comportement, 1979.

Statistique Canada, Recensement 1976, Ottawa, 1982.

TESSIER, M., Sexualité et prévention: d'abord l'affaire des jeunes, Montréal, Bureau de consultation jeunesse, 1985.

ThéвAult, M., Grossesse et adolescence; élaboration d'une stratégie globale d'intervention, Montréal, Département de santé communautaire du Centre hospitalier Saint-Luc, 1985.

WILKINS, J., "La sexualité durant l'adolescence : phénomène pluridimensionnel ", dans: Jean WiLkins et al., Médecine de l'adolescence: une médecine spécifique, Montréal, Centre d'information sur la santé de l'enfant/Promotion, Hôpital Sainte-Justine, 1985.

ZeLNIK, M. et J.F. KANTNER, "Sexual activity, contraceptive use and pregnancy among metropolitan area teenagers : 1971-1979 ", Family Planning Perspectives, vol. 12, n० 5, 1980 : 230-237. 